\title{
A global effort to understand the riddles of COVID-19 and cancer
}

\author{
Cancer has found a formidable foil in COVID-19, and this has brought to the fore the early concerns that COVID-19 \\ could have a deeper impact on oncology patients. Two studies now provide insights into the enigma surrounding \\ the determinants of the worsening of COVID-19 symptoms in patients with cancer.
}

\author{
Vivek Subbiah
}

\section{evere acute respiratory syndrome coronavirus 2 (SARS-CoV-2) and its associated coronavirus disease} 2019 (COVID-19) have brought an unprecedented level of disruption globally. Every oncology stakeholder, especially patients, families and clinicians, face reconciling the particular challenges brought on by the intersection of these two life-threatening diseases. Two studies in Nature Cancer, by Albiges et al. ${ }^{1}$ and Rugge et al. ${ }^{2}$, provide key insights into the determinants of the outcome of severe COVID-19 in patients with cancer.

The understanding of COVID-19 relies heavily on the timely dissemination of knowledge arising from the most impacted areas. Initially faced with the critical need for guidelines on the delivery of cancer care in the context of COVID-19, expert panels from oncology societies and health ministries examined emerging data from Asia to issue recommendations and enable oncologists and patients navigate the pandemic while balancing the risks and benefits of specific cancer-directed care ${ }^{3-5}$.

The prevailing concerns for oncologists and patients with cancer alike center on cancer diagnostics, as well as cancer-specific clinical and treatment characteristics, and the risk of negative outcomes concurrent with COVID-19. The earliest studies of this topic demonstrated conflicting findings. Specifically, one early report from New York City analyzed a cohort of 5,688 patients with COVID-19, of whom 334 (6\%) also had cancer. Although a higher frequency of endotracheal intubation was noted among those with cancer (relative risk $=1.89$; $95 \%$ confidence interval $(\mathrm{CI})=1.37-2.61$ ) without stratification by age, this series did not find a significant difference between the patients with cancer and those without cancer, in their mortality ${ }^{6}$. In contrast, another multi-center study of 14 hospitals in the Hubei province of China enrolled 641 patients with COVID-19 (105 with cancer and 536 without, matched by age and by time and site of hospitalization) and showed that those patients with cancer and COVID-19 had a higher risk of negative outcomes, including in-hospital infections, intensive care unit admissions, invasive mechanical ventilation and observed death rates ${ }^{7}$. Overall, the inconsistent early findings from these and other series highlighted the need for continued data reporting on mortality and other clinical outcomes of patients with both cancer and COVID-19.

To that end, Albiges et al. ${ }^{1}$ and Rugge et al. ${ }^{2}$ presented their early independent studies from France and Italy, in order to add to this growing body of evidence. In their study, Albiges et al. analyzed variables with prognostic value for clinical worsening or death in 178 patients with cancer and COVID-19, seen over a six-week period at the Gustave Roussy Cancer Center ${ }^{1}$. The investigators in this study reported an increased risk of death among patients over 70 years of age, current or former smokers, an Eastern Cooperative Oncology Group performance status of 2 or worse at the last follow up visit, presence of metastatic disease, and/or receipt of cytotoxic chemotherapy in the past three months. A comparison with regional data showed that the mortality rate of $17.4 \%$ among this cohort of patients was similar to that of contemporaneously admitted patients in the Paris area. One of the most important findings of the study by Albiges et al. ${ }^{1}$ is the glimpse it provides of the impact of COVID-19 on cancer strategies and post-COVID-19 assessment of patients with cancer, which was not readily provided by the other large registry studies. Through preliminary patient-reported outcomes (PROs), the authors offered a rough recall assessment of what 146 patients with cancer and COVID-19 experienced in the first month assessment; the study highlighted the finding that the PROs included 75\% asthenia, $42 \%$ exertional dyspnea and $30 \%$ cough. These rough estimates had a recall bias, and future studies should include validated PRO tools to improve the rigor needed to result in evidence-based guidelines. Objective lab-based measures can offer hints about the severity of disease and determinants of acute mortality and morbidity, but validated PRO tools at multiple time points can indicate determinants of long-term morbidity experienced by patients. COVID-19 may leave a long-lasting impact into the mental health of patients with cancer (and their caregivers), in addition to the physical toll, and such PRO measures should capture those outcomes in detail as well.

In the study by Rugge et al., the researchers presented a population-level view of COVID-19 and cancer among residents from the Veneto region ${ }^{2}$. This study was important, as most of the studies reported from major registries were derived mainly from patients admitted to hospitals, whereas not many studies have reported data directly from the community. Hence, population-based, clinico-epidemiological studies are important for reflecting COVID-19 in specific populations with cancer that are better representative of the general population with cancer. The investigators reported here the prevalence of cancer (either current or former) among the 84,246 patients consecutively tested for SARS-CoV-2; additionally, they explored an association between a prior diagnosis of cancer and COVID-19-related clinical outcomes. The main finding of this report was that $5.7 \%$ patients $(4,789$ of 84,246$)$ had previously been diagnosed with cancer, and among the SARS-CoV-2-positive patients, $7.8 \%$ had a diagnosis of cancer (723 of 9,275 ). Intriguingly, prevalence of cancer was not associated with risk of infection (odds ratio $=0.97 ; 95 \% \mathrm{CI}=0.89-1.06$ ). However, the proportion of people with COVID-19 who were hospitalized was 
higher among patients with cancer (56.6\%) than among other people (34.4\%), and so was the proportion of mortality $(14.7 \%$ versus $4.5 \%$, respectively). In addition, older adults 70 years of age and above, male patients, and those patients whose cancer had developed no more than two years before they became COVID-19 positive had an increased risk of adverse outcomes. While the study in Veneto did not identify an association between a history of cancer and SARS-CoV-2 infection, it did report increased risk of mortality ${ }^{2}$. Being a clinico-epidemiological study, its data may show trends in a particular region for crisis-management purposes, and similar studies in distinct areas should be designed in order to validate these findings.

Now that there are data from large registries of COVID-19 in patients with cancer, such as CCC19 (the COVID-19 and Cancer Consortium) ${ }^{8}$, UKCCMP (the UK Coronavirus Cancer Monitoring Project) ${ }^{5}$ and TERAVOLT (Thoracic Cancers International COVID-19 Collaboration) ${ }^{9}$, these data should be compared, as similarities and differences found may help in the future care of patients with cancer (Fig. 1). A recent pooled analysis of 52 studies, comprising 18,650 patients with both COVID-19 and cancer, showed that the probability of death was $25.6 \%$ (95\% CI $=22.0-29.9 \%$; $\mathrm{I}^{2}$ (heterogeneity statistic $)=48 \cdot 9 \%)^{10}$. Increasing age seemed to be consistently associated with death in the Gustave Roussy cohort ${ }^{1}$ and Veneto data $(\geq 70 \text { years of age })^{2}$, in patients at Institut Curie hospitals (the 'Curie cohort') ${ }^{11}$, in the CCC19 registry $^{8}$ and in the TERAVOLT registry ${ }^{9}$. One of the main advantages of single-center studies, such as the one from Gustave Roussy ${ }^{1}$, is that they provide a granular and deep 'dive' into the lab abnormalities of patients with COVID-19 and cancer. In the Gustave Roussy study, nonspecific inflammatory markers, such as a C-reactive protein concentration of over $50 \mathrm{mg} / \mathrm{ml}$ and a lactate dehydrogenase concentration of $250 \mathrm{IU} / \mathrm{L}$, were substantially associated with increased risk of clinical worsening, whereas a C-reactive protein concentration of over $50 \mathrm{mg} / \mathrm{ml}$ and $\mathrm{D}$-dimer values of $>3 \mu \mathrm{g} / \mathrm{l}$ were associated with an increased risk of death ${ }^{1}$.

None of the studies of COVID-19 and cancer, including the large registry studies, reported on the kinetics of specific testing indicative of the severity of COVID-19. Monitoring of temporal profiles and serial monitoring of viral loads from various tissue samples, viral shedding, and titers of IgM and IgG antibodies in patients with cancer may improve understanding of the humoral immune response after SARC-CoV-2
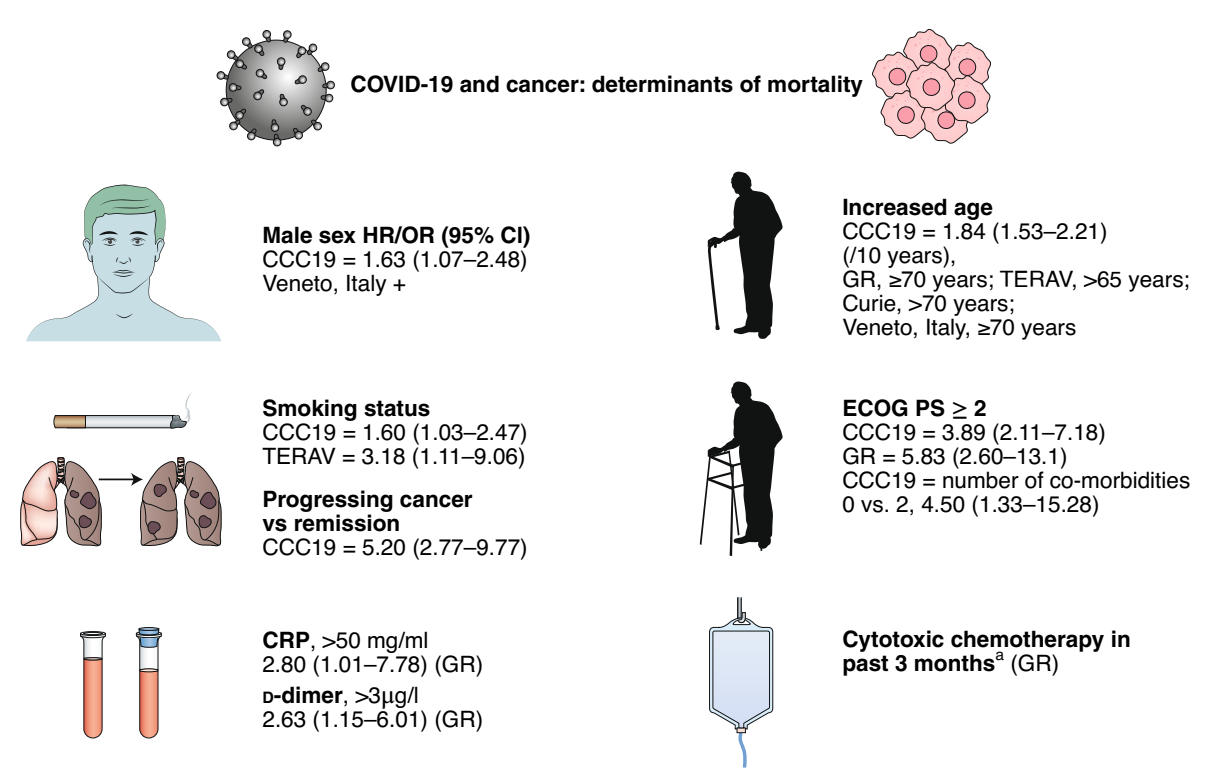

Cytotoxic chemotherapy in past 3 months ${ }^{\mathrm{a}}$ (GR)

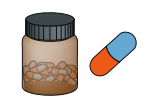

AZ + HCQ Rx CCC19 = $2.93(1.79-4.79)$

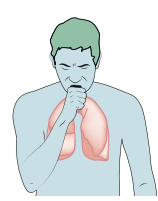

Dyspnea $^{\mathrm{a}}$

(UKCCMP; TERAV)

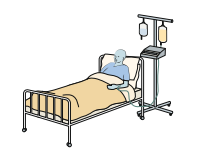

ICU stay ${ }^{\mathrm{a}}$

(UKCCMP; Veneto, Italy)

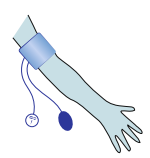

Hypertension $^{\text {a }}$ (Curie)

CoVID19 severity score ${ }^{a}$ (UKCCMP)

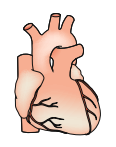

Cardiovascular disease $^{a}$ (UKCCMP)

Fig. 1 | Determinants of mortality for COVID-19 and cancer from published studies. Summary of selected factors that determine worse outcomes for COVID-19 and cancer, derived from the univariate and multi-variate analyses of COVID-19 studies of the CCC19 registry ${ }^{8}, \mathrm{UKCCMP}^{5}$, Gustave Roussy cohort (GR)', the TERAVOLT registry (TERAV) ${ }^{9}$, the Curie cohort ${ }^{11}$ and the Veneto region of Italy ${ }^{2}$. Factor values and hazard ratio/odds ratio values (HR/OR; partially adjusted odds ratio for CCC19) $(95 \% \mathrm{CI}$ values in parentheses) are provided for multivariate analysis of prognosis factor of death. aUnivariate analysis only. Note that many registry studies have selection bias from reporting only symptomatic patients with COVID-19 and cancer who were admitted to the hospital and may not reflect data from asymptomatic patients with COVID-19 and cancer. Moreover, there is a lack of control for patients with COVID-19 without a diagnosis of cancer. CRP, C-reactive protein; AZ + HCQ Rx, azithromycin plus hydroxychloroquine treatment; ICU, intensive care unit; ECOG PS, Eastern Cooperative Oncology Group performance status. Created with BioRender.com.

infection, as well give more insight into the prognosis of patients. Moreover, this may expand the understanding of treatment planning for the use of convalescent plasma or specific anti-viral therapies and vaccine development and scheduling administration for patients with cancer.

The unprecedented global challenge that the COVID-19 pandemic imposes on cancer care has highlighted inherent systemic limitations in technology, funding and infrastructure, although global efforts in clinical reporting have contributed to fighting these two diseases simultaneously. What seems essential is a robust global data infrastructure that enables an even faster dissemination of key findings and data sharing. The structure of data collection in 'silos' seems to be ad hoc, with several of the registries from grassroots movements having no central funding ${ }^{12}$. Lessons learned from this pandemic should include first 
the necessity of an international registry for data science, bio-surveillance and an infrastructure with funding to conduct multi-arm master clinical trials using shared control arms with ease. Second, future data collection should focus on attention to details, including PROs, both acute and chronic. Third, studies of COVID-19 in patients with cancer should start reporting viral loads and other immunological parameters, if available. Fourth, the rapidity of reporting should not compromise the accuracy, veracity and validity of data. All studies should ensure they have appropriate regulatory approval and have mechanisms for sharing de-identified data, and they should be source-verifiable and auditable by independent third parties. Fifth, there is a need to report all patients with COVID-19 and cancer, including patients who are admitted to the hospital plus those who are not admitted (who may be less sick and asymptomatic) and to compare datasets of patients with COVID-19 without cancer as a contemporaneous control population. Sixth, efforts should be made to also collect data from pediatric patients with cancer who have COVID-19. Seventh, more systematic collaboration is needed among clinical researchers, epidemiologists and basic/translational researchers in elucidating the fundamental mechanisms and immunobiology of COVID-19 in patients with cancer. Finally, with the growing body of evidence of the effects of COVID-19 on patients with cancer, it may be time to reflect, re-visit, revise and re-work the guidelines put forth by major cancer organizations and amend their recommendations as more prospective evidence accumulates. To confront COVID-19 in patients with cancer is to encounter two parallel threats. Nonetheless, these global efforts offer a reason to advance understanding of the huge gaps in knowledge of these two life-threatening diseases.

The studies by Albiges et al. ${ }^{1}$ and Rugge et al. ${ }^{2}$ support the early concern on the clinical frontlines that COVID-19 may have a deeper than anticipated impact on patients with cancer than on otherwise healthy adults, and they present valuable insights into the questions about the observed worsened outcomes in this vulnerable group of patients.

\section{Vivek Subbiah}

Department of Investigational Cancer Therapeutics, Division of Cancer Medicine, University of Texas MD Anderson Cancer Center, Houston, TX, USA.

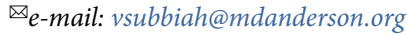

Published online: 9 October 2020

https://doi.org/10.1038/s43018-020-00129-w

References

1. Albiges, L. et al. Nat. Cancer https://doi.org/10.1038/s43018-02000120-5 (2020).

2. Rugge, M., Zorzi, M. \& Guzzinati, S. Nat. Cancer 1, 784-788 (2020).

3. Alhalabi, O. \& Subbiah, V. Trends Cancer 6, 533-535 (2020)

4. Alhalabi, O., Iyer, S. \& Subbiah, V. EClinicalMedicine 23, 100374 (2020).

5. Lee, L. Y. W. et al. Lancet 395, 1919-1926 (2020).

6. Miyashita, H. et al. Annals Oncol. 31, 1088-1089 (2020).

7. Dai, M. et al. Cancer Discov. 10, 783-791 (2020).

8. Kuderer, N. M. et al. Lancet 395, 1907-1918 (2020).

9. Garassino, M. C. et al. Lancet Oncol. 21, 914-922 (2020).

10. Saini, K. S. et al. Eur. J. Cancer 139, 43-50 (2020).

1. Vuagnat, P. et al. Breast Cancer Res. 22, 55 (2020).

12. Desai, A. et al. Nat. Cancer 1, 473-476 (2020).

Competing interests

The author declares no competing interests. 